\title{
LoRaWAN for Wind Turbine Monitoring: Prototype and Practical Deployment
}

\author{
Konstantin Mikhaylov \\ Centre for Wireless Communications \\ University of Oulu \\ Oulu, Finland \\ konstantin.mikhaylov@oulu.fi
}

\author{
José Manuel Martín Rapún \\ Instrumentacion y Componentes S.A. \\ Zaragoza, Spain
}

\author{
Abdul Moiz \\ Centre for Wireless Communications \\ University of Oulu \\ Oulu, Finland \\ abdul.moiz@oulu.fi
}

Sergio Ayuso Gascon

Instrumentacion y Componentes S.A. Zaragoza, Spain

\author{
Ari Pouttu \\ Centre for Wireless Communications \\ University of Oulu \\ Oulu, Finland \\ ari.pouttu@oulu.fi
}

\begin{abstract}
The energy grids became a cardiovascular system of the today's civilization. In the recent years the new political initiatives aiming at reducing the pollution by increasing the share of energy generated by renewables have gained momentum, which introduces novel challenges and use cases. In the paper we investigate the utility and report our experiences of deploying a prototype wind-turbine monitoring solution based on the recently developed low power wide area network (LPWAN) technology named LoRaWAN. We engineer, instrument and deploy the complete end-to-end solution, starting from the power generation monitoring sensor node and up to the IoT platform with a web-based graphical user interface. The details of the design and deployment choices, and the results and lessons learned coming from analysis of operation of the deployed system over the ninemonth period are reported. These results show that LPWANs in general and LoRaWAN technology in particular is suitable for monitoring the energy infrastructure.
\end{abstract}

Keywords - Electric Grid, Smart Grid, LPWAN, LoRaWAN, monitoring, wind turbine, use case, application, experiment

\section{INTRODUCTION}

The recent decades have dramatically changed the face of the world and the human lives with respect to many aspects. The advancements of the information and communication technologies were one of the key drivers for this remarkable transformation. Notably, this metamorphosis is far from being finished - the new technologies and concepts constantly bring along with them novel opportunities.

The electric power generation and distribution network, which became over the past half a century the cardiovascular system of the today's civilization, has received a limited attention so far. On one hand, this is caused by the ultra-high cost of a mistake - why to take a risk of changing something already working for decades? On the other hand, there are challenging requirements: the huge span and localization of the grid elements as well as the economic feasibility. Nonetheless, the recent advancements with respect to the Machine-to-Machine (M2M) wireless communication technologies and namely the recently introduced Low Power Wide Area Network (LPWAN) technologies can enable to change this situation and contribute to converting the conventional energy grid into the Smart Grid [1],[2].

The attempts to couple the distribution and generation grids with wireless communication are far from being novel. The residential power meter solutions based on ZigBee [3][4], as well as on the other wireless communication technologies [5],[6], have already been designed and their utility was investigated. The distributed energy generation and consumption balancing using wireless communication

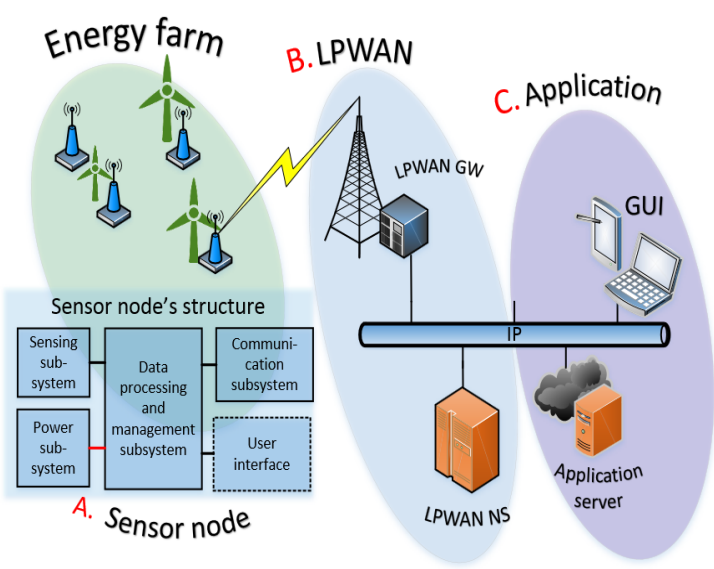

Fig. 1. High-level architecture of the proposed solution.

technologies has been comprehensively addressed, e.g., within the EU H2020 P2PSmarTest project ${ }^{1}$, and in [7][8]. The possibility of the interaction between the smart grids and the next generation communication infrastructure, as well as of the energy trading within regional grid, was discussed in [9]. Quite recently, a number of wireless monitoring solutions for energy generation from renewables have been introduced, e.g., in [10]-[13]. In [14] the authors discuss also the techno-economic aspects of using wireless technologies compared to wired technologies for monitoring renewable generators, concluding that wireless solutions may enable substantial savings, especially for big deployments in the countries with high labour prices.

As can be seen from, e.g., [10]-[13], the state-of-the-art energy generation monitoring solutions of today are based on such conventional wireless communication technologies as IEEE 802.15.4 (ZigBee) and IEEE 802.11 (WiFi). Nonetheless, the recent years have brought to the market new technologies, which may appear to be more suitable for power generation monitoring. Therefore, in this paper we explore the possibility and utility of instrumenting a renewable monitoring solution based on an LPWAN technology named LoRaWAN. We report the details of design and the results of practical deployment and utilization of a complete system, starting from the sensor and up to the user application. The presented results on one hand confirm the feasibility of using LPWANs for energy generation monitoring, and on the other - show some of the limitations to be addressed in future. Therefore, the results, reported in this paper, can be valuable and serve as a reference for the readers from Academy and Industry.

${ }^{1}$ P2PSmarTest project web page: http://www.p2psmartest-h2020.eu/ 


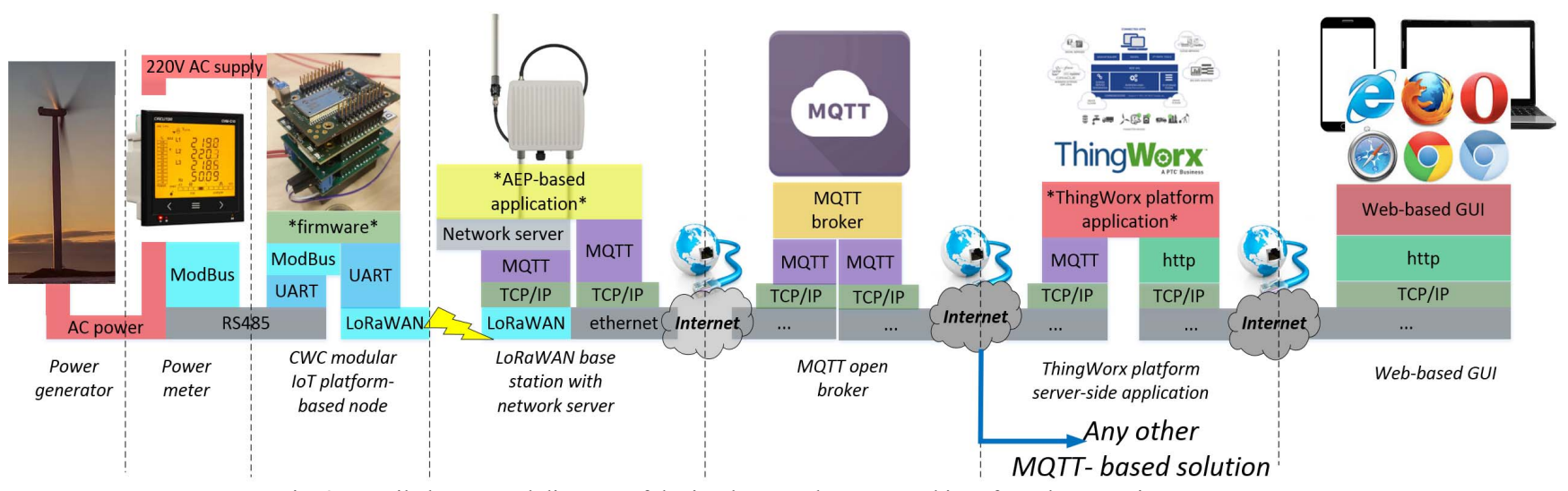

Fig. 2. Detailed structural diagram of the implemented system and interfaces between its components

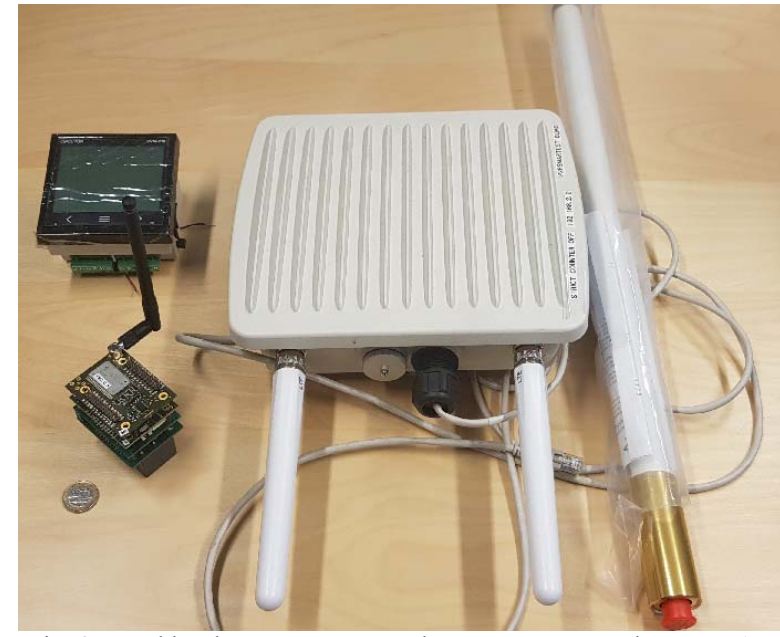

Fig. 3. Used hardware: a sensor node, power meter and LoRaWAN gateway with $868 \mathrm{MHz}$ antenna (in plastic bag)

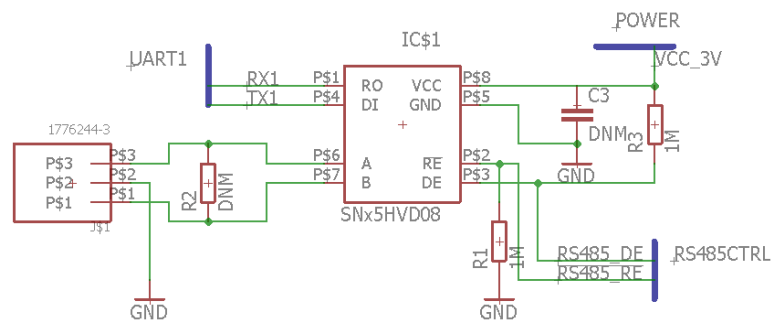

Fig. 4. Schematic of the designed RS485/422 UART converter board

\section{SYSTEM ARCHITECTURE AND COMPONENTS}

\section{A. Requirements and Approaches}

Today there is a significant push for increasing the amount of the "green" energy generated. In the European Union, this is driven by the "."20-20-20" strategy, which targets reduction of the greenhouse gas emissions, increase of the energy generated from renewables, and improvement of the energy efficiency by $20 \%$ each by $2020^{2}$. The initiatives to increase the energy generated from renewables are also taken in other countries and regions around the globe, including the USA, China, Australia, Gulf region, etc.

This is one of the major reasons for the constant increase for both the number and the size of wind and photovoltaic

\footnotetext{
${ }^{2}$ European CommissionEnergy, Climate change, Environment, https://ec.europa.eu/clima/policies/strategies/2020 en
}

pharms deployed ${ }^{3}$. In order to ensure efficient and fault-free operation of these systems, an effective solution enabling monitoring of each single generator is required. Among the key requirements for such a monitoring system are:

- possibility of deployment in remote geographical areas, including the ones where the conventional communication infrastructure is poor or absent (e.g., offshore wind farm deployments),

- $\quad$ significant geographical span of the area of interest (units to dozens kilometres) and various deployment shapes (grid, line, etc.),

- $\quad$ good scalability, since a contemporary farm may compose hundreds to thousands generators, each equipped with multiple sensors,

- reasonable combination of the device cost, operational expenditures, energy consumption and the provided service of quality.

These requirements can be addressed within two different architectures. The former one is the self-configurable ad-hoc network. The second option is a centralized network operating in unlicensed frequency bands and deployed as a private network. This deployment option is supported by several state-of-the-art LPWAN technologies, with LoRaWAN being one of the most well-known ones. The LPWAN-based solution results in a simpler and easily manageable architecture with, potentially, more uniform distribution of the energy consumption between the various nodes. Therefore, in what follows, we focus specifically on this option, and investigate by the means of practical experiment the feasibility, and study the utility of implementing and utilizing a solution based on this approach.

\section{B. System Architecture and Key Components}

The high-level architecture of our proposed solution for energy generation monitoring is depicted in Fig. 1. The envisaged system consists of the three major components, namely the sensor devices, the LPWAN gateway and data backbone, database and visualization solution.

The sensors devices are deployed right on top or around the power generators, measuring the parameters of interest, such as: the voltage, current, phase and other quality parameters of the generated energy, infrastructure parameters like the temperature and vibration, and the various environment parameters (e.g., speed of wind, humidity, and

\footnotetext{
${ }^{3}$ Refer, e.g., to https://www.4coffshore.com/windfarms/
} 


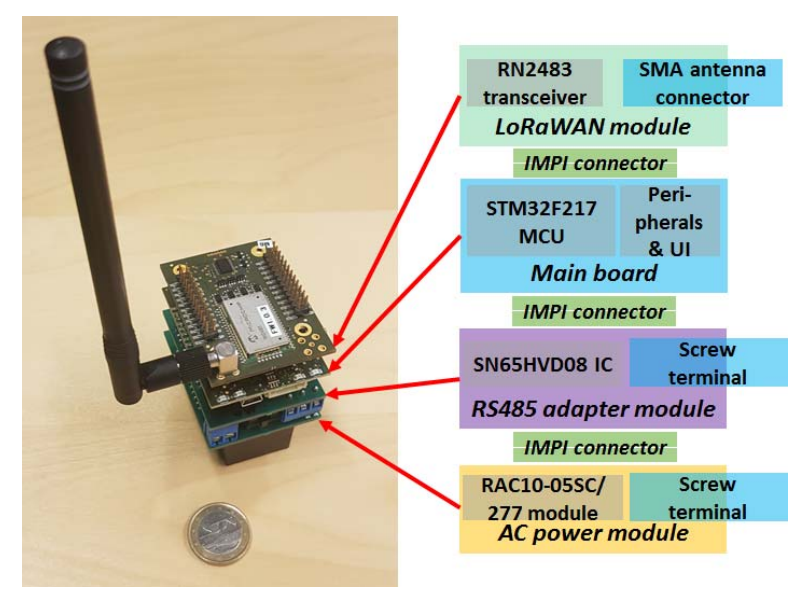

(a)

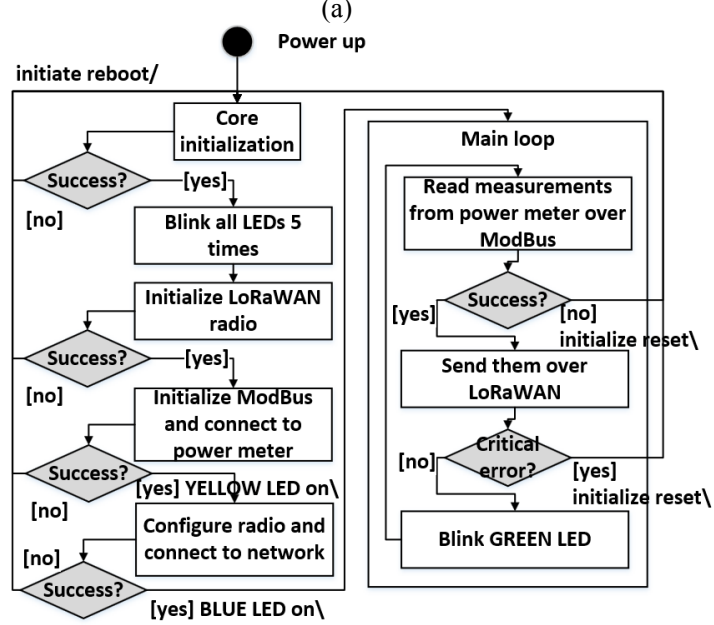

(b)

Fig. 5. Sensor node: (a) photo and the structure of implemented sensor node, (b) algorithm of sensor node's firmware operation

light conditions). Depending on the design of the generators, the sensor nodes may be powered with energy harvesting, batteries or even mains. In the case if the generator is already equipped with a monitoring system - the need for interfacing the sensor nodes and the default monitoring system arises.

A typical LPWA network [15], such as, e.g., LoRaWAN, is built in a star-of-stars fashion. One or multiple gateways (GWs) convey the traffic between the sensor nodes and the network server (NS). A conventional LPWA GW is built around a Linux-controlled industrial computer enriched with the necessary radio communication capabilities and utilizing an internet protocol-based backbone communication interface. Depending on the implementation, the backbone can operate over a wired (e.g., Ethernet) or wireless (e.g., LTE or WiFi) physical channel. The single NS manages the data transfers from and to multiple GWs, and handles the exchange of data with applications over specific interfaces.

Finally, the third key component is a subsystem responsible for processing the data, logging them and presents the relevant ones to an end user. Depending on the target audience and the desired performance and features support, a very broad range of technical solutions can be utilized at this stage.

\section{Implementation: Hardware and Software Components}

The detailed system-level diagram of our implemented system is depicted in Fig. 2 and the devices used in our deployment are shown in Fig. 3. In what follows each of them is discussed separately. Note, that since our system is planned to be interfaced with the already existing turbine monitoring system, the need for supporting backward compatibility has affected our designs.

\section{1) The End Device}

The end device for our proof-of-concept deployment has been constructed using the modular IoT hardware platform developed at the CWC [16]. In the core of the node lies processing and power module built around a STM32F217 32-bit Core-M3 ARM microcontroller. The LoRaWAN radio module hosting a commercial RN2483 transceiver is stacked on top of the main board. On the bottom, two other modules, which were designed specifically for this deployment, are attached. The lower one features an AC-DC converter and enables powering the sensor node directly from mains.

The RS485 and RS422 interfaces are often utilized by the commercial industrial-grade wired monitoring solutions, such as power meters. Even though the communication protocol is similar to that of conventional universal asynchronous receiver-transmitter (UART), the need for operating with differential signaling and the higher voltage levels requires use of a converter, which is implemented in the adapter module. The schematic of the adapter board is shown in Fig. 4, and the photo of the node and its structural diagram are presented in Fig. 5 (a).

The sensor node is interfaced with the CVM-C10 power meter from CIRCUTOR S.A., which is already deployed at a wind turbine and which can measure over one hundred power-related parameters. The communication between the sensor node and the power meter is handled through the designed RS485 adapter board using the Modbus protocol.

The high-level algorithm of the sensor node's operation is depicted in Fig. 5 (b). On power-up the sensor node initializes and activates the LoRaWAN transceiver, and establishes the connection to the power meter, and then periodically reads the parameters of interest, such as the currents $(\mathrm{A} 1, \mathrm{~A} 2, \mathrm{~A} 3)$ and voltages $(\mathrm{V} 1, \mathrm{~V} 2, \mathrm{~V} 3)$ for the three phases, the active and inductive three-phase power (kWIII and kvarLIII, respectively), and the three-phase power factor (PFLIII). These data are further packed into a radio packet and provided to the LoRaWAN transceiver for transmission.

The similar solution may have been instrumented using any other contemporary embedded platform. Under mass production, a specialized integrated board hosting all components may provide a more cost-efficient solution compared to the use of modular-based system.

\section{2) The LoRaWAN Gateway and Network Server}

To enable LoRaWAN communication for the test area we deployed a MultiConnect ${ }^{\circledR}$ Conduit ${ }^{\mathrm{TM}}$ commercial LoRaWAN GW from MultiTech. The GW was selected due to three major reasons. First, it is implemented in IP67 package and is equipped with $868 \mathrm{MHz}$ band daughter card. Second, the LoRaWAN NS is deployed right on the GW. Third, the GW features two possibilities for implementing backbone connectivity, namely LTE and Ethernet. In our deployment, due to the cost and availability reasons the latter is utilized. The power is supplied to the GW also through the Ethernet cable. 


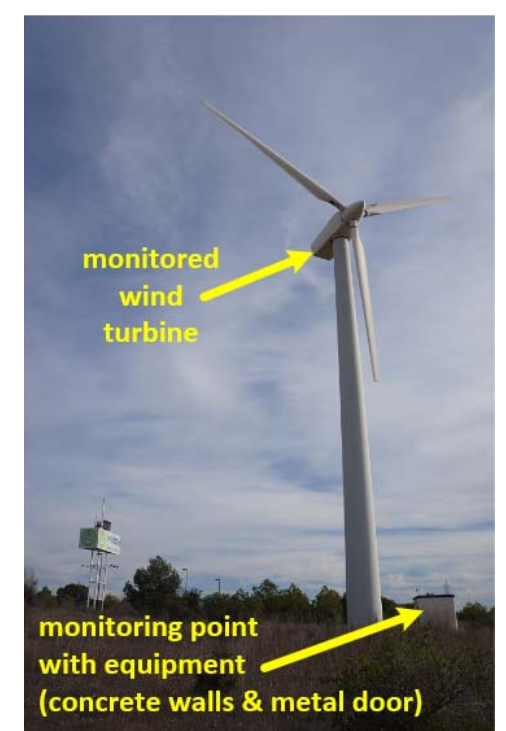

(a)

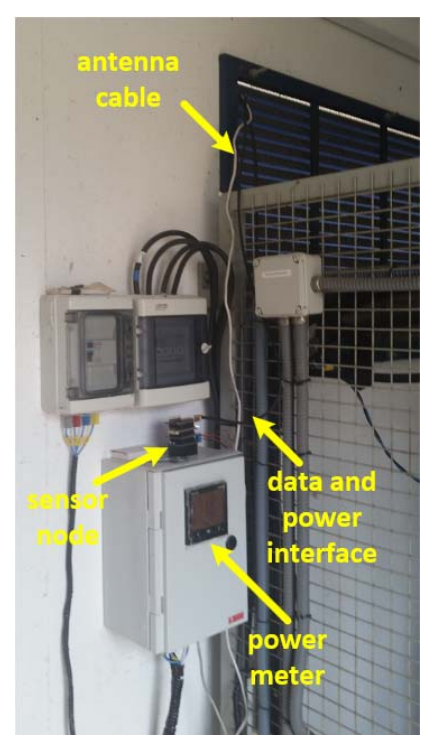

(b)

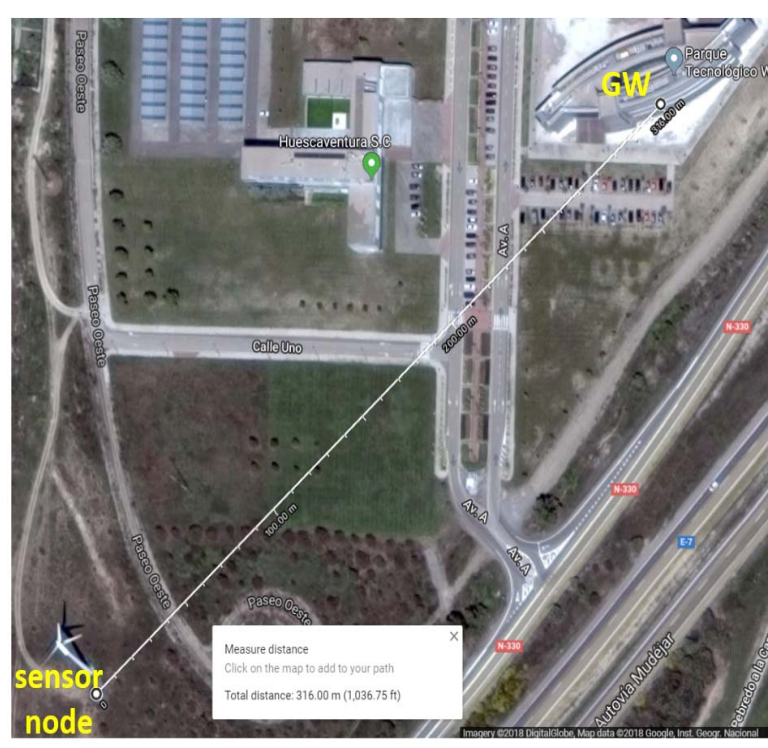

(c)

Fig. 6. Deployment (a) wind turbine (b) connection of the sensor node in the monitoring point, (c) Google Maps satellite view of the deployment area

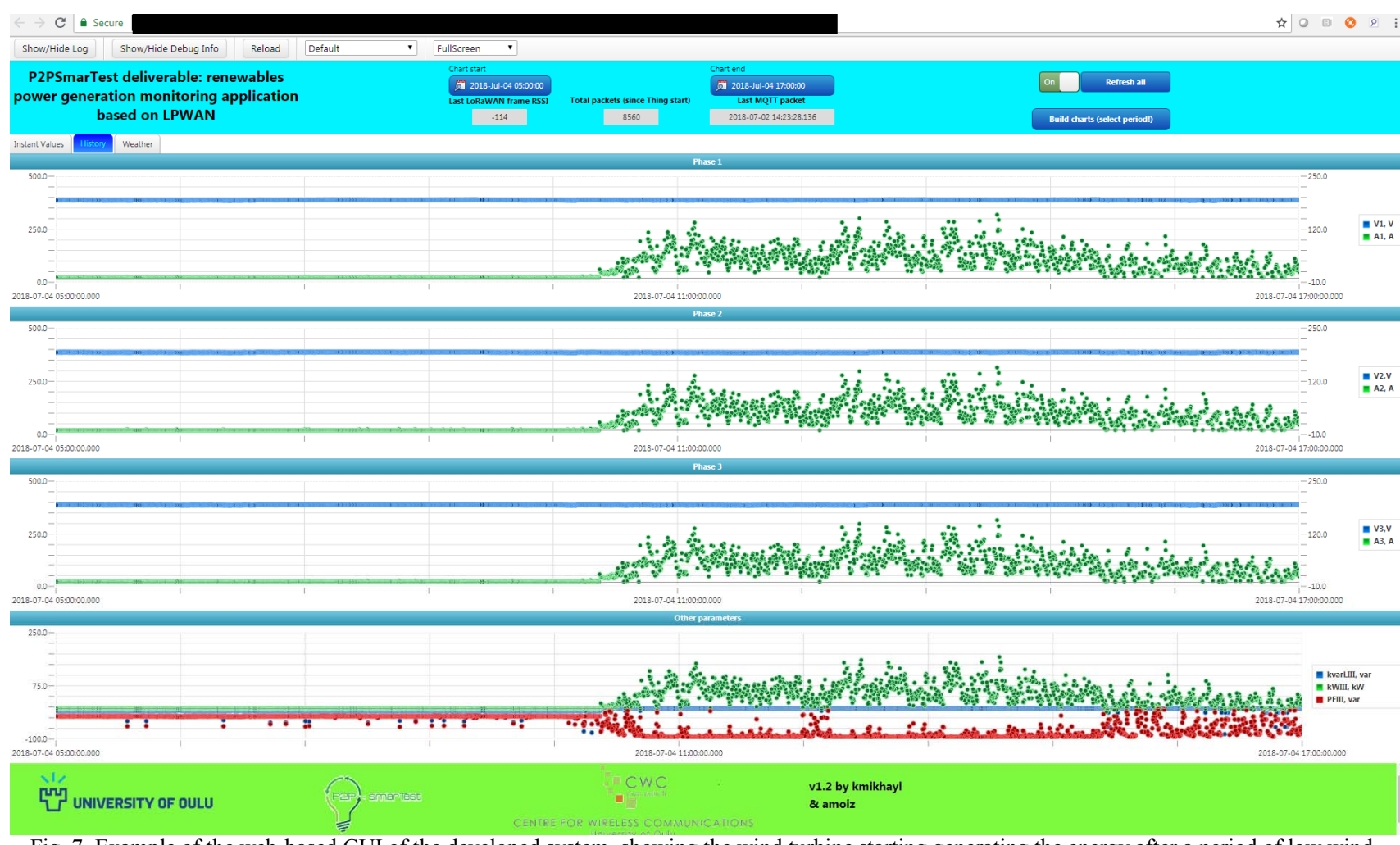

Fig. 7. Example of the web-based GUI of the developed system, showing the wind turbine starting generating the energy after a period of low wind

On top of the NS by using the Node-RED tool we have deployed a proprietary application. The application receives from the NS all the LoRaWAN packets, validates them and adds the service information, and sends via Message Queuing Telemetry Transport (MQTT) protocol over backbone connection to a third party MQTT broker over secure connection. The MQTT protocol was selected due to the ease of its implementation and handling. Alternatively, CoAP or pure TCP/UDP connection may have been used.

\section{3) The MQTT broker}

The MQTT broker (connectable through TLS/SSL connection) acts as the mediator for the sensor data. The NSbased application connects to MQTT broker and uses MQTT publish mechanism to deliver each new report received from the sensor to specific topic. The other interested parties (e.g., an IoT platform) can subscribe to the same topic and start receiving the publications sent by the LoRaWAN NS. In our deployment we used a commercial MQTT broker solution to ease the deployment and management of the system.

\section{4) IoT Platform}

For analyzing the data and implementing the graphical user interface (GUI) the ThingWorx platform was used. The platform was selected due to ease of its use and broad functionality provided. The MQTT "thing" connects to the MQTT broker and subscribes to the topic of interest. After that, the change of the MQTT thing parameters happens each time the new data are reported by the GW. All the parameter changes are logged by the platform to a database. 


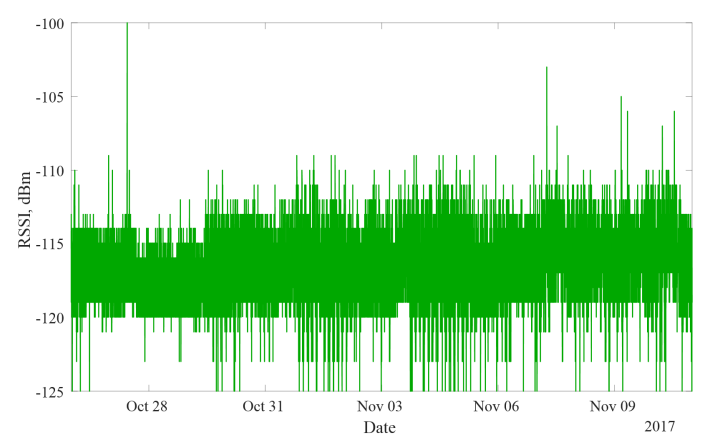

(a)

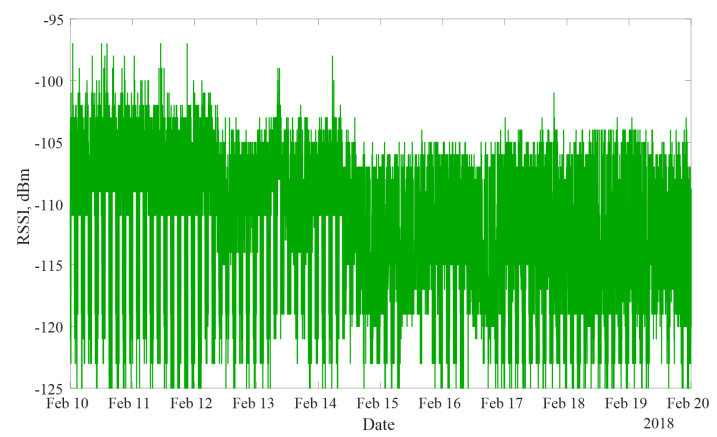

(c)

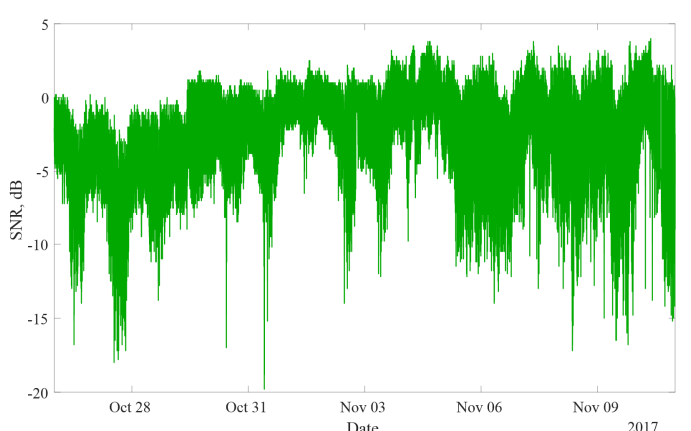

(b)

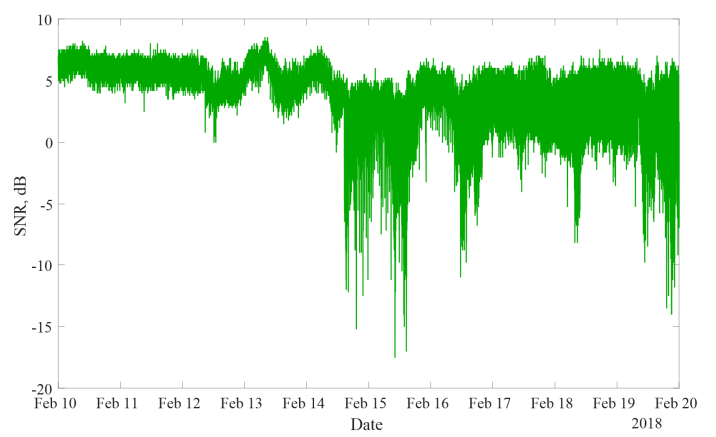

(d)

Fig. 8. RSSI and SNR for two selected periods

The web-based GUI enabling visualization of the most recent measurements, as well as of the historic data was designed using the means of ThingWorx (i.e., the "mashup" tools). Also the ThingWorx application was configured to connect to an external weather service and log the current weather conditions near to the site, where the monitored windmill is installed. This provides valuable context information helping to make sense of the measurements.

\section{DEPLOYMENT AND CONFIGURATIONS}

In October 2017 one of the test wind turbines in WALQA technology park, Huesca, Spain, has been instrumented with the developed system. Figs. 6 (a) and 6 (b) illustrate the turbine and its environment, and show the connection of the sensor node with the rest of the monitoring equipment, respectively. Fig. 6(c) presents the satellite view of the deployment area and the locations of components.

As can be seen from Fig. 6 (b), the sensor node was installed in the area, storing all the monitoring and power control equipment related to the turbine. The sensor node is connected to the power meter's data lines in parallel with the already deployed WiFi-based monitoring solution and is powered from the same power rails as the power meter. The initial experiments have shown that the concrete walls of the monitoring point block the radio signal if the antenna is located inside. Therefore, the antenna was taken out to the roof, and connected to the node with a cable.

Originally, the GW was planned to be installed on the roof of the four-floor park management building, but then we experimentally find out that even when installed indoors on the first floor next to the window the GW can receive the radio signals from the sensor node. The GW was powered through Ethernet, which was also used for providing the backbone connectivity for the GW (i.e., for communication to the MQTT broker).
The sensor node was configured to operate with spreading factor of 12 and use the maximum transmit power of $14 \mathrm{dBm}$. This was done since the deployed device is powered from mains and thus is not energy-limited. Given that no downlink is used, our private LoRaWAN network was configured to use in addition to the default channels also the $869.5 \mathrm{MHz}$ band (h1.6 band according to ERC 70-03), enabling transmission with up to $10 \%$ duty cycle. The reporting period of a sensor was set to 20 seconds with additional 0-10 second random component. Given the limited scale of our installation, this configuration makes sense, albeit the further increase of the number of devices would require revising it. Neither adaptive data rate, nor acknowledgements have been used. For connecting to the GW the activation by personalization procedure was utilized.

\section{RESULTS}

First, the basic functionality of the system as whole and of its individual components was validated. Fig. 7 illustrates the exemplary GUI of our developed system for 12 hours period. All nine parameters of interest are delivered from the sensor node to the IoT platform and visualized, enabling to monitor in sufficient details the dynamics of the wind turbine's operation. The data can also be downloaded from the IoT platform and used for the offline analysis.

To this day, the system has been up and running for about 9 month, collecting over these period half a million reports. To get a better insight into the utility of LoRaWAN for powering monitoring scenarios, we have processed these data to assess the selected key communication performance indicators [17], namely the reliability, latency, and cost.

When it comes to the reliability of the deployed system, our results are somewhat controversial. On one hand, throughout the analyzed period there were few periods, when no data was recorded to the IoT platform. The further studies have shown that the problem is primarily caused by the 
absence of communication between the MQTT broker and the IoT platform. At the very same time, the analysis of the periods, for which the data is available, show that in average more than $96 \%$ of the radio packets were received correctly. Even though, there were few periods with several dozens packets lost in a row. Note, that given the complex structure of our system, this may have been caused by multiple reasons over which we have limited control (e.g., power outage, service or internet connection issues at the deployment site, MQTT broker or IoT platform's servers).

Fig. 8 illustrates the variation of the Radio Signal Strength Indicator (RSSI) and Signal-to-Noise Ratio (SNR) for the packets received by the LoRaWAN GW for two selected time intervals. The mean and standard deviation of RSSI for the depicted cases is $-117 \mathrm{dBm}$ and $1.8 \mathrm{dBm}$ for the first interval, and $-113 \mathrm{dBm}$ and $5 \mathrm{dBm}$ - for the second interval. The mean SNR was $-4 \mathrm{~dB}$ for the first and $2.6 \mathrm{~dB}$ for the second interval. Throughout the whole campaign, the mean RSSI was $-115 \mathrm{dBm}$ and mean SNR $-0.7 \mathrm{~dB}$. As one can see, there were both long and short-term fluctuations in the radio channel. Note that the sensitivity level for LoRaWAN transceivers is in the order of $-148 \mathrm{dBm}$, which gives a channel budget of about $30 \mathrm{~dB}$ thus enabling substantial increase of communication range.

Analyzing the end-to-end latency, we noted that the average time required for delivering the data from a sensor node to the IoT platform was about 30 seconds. Occasionally, significant fluctuations in the delivery times were observed. This can be explained due to the complex structure and geographic remoteness of the components (GW - Spain, MQTT broker - Ireland, IoT server - Finland).

Finally, with respect to the cost, LoRaWAN-based solution appeared to be quite low cost. Based on the analysis of the data bases of the component providers, the price of a LoRaWAN transceiver is today in the order of several EUR, which makes it $25-50 \%$ more expensive than ZigBee, and more than twice cheaper than the cellular radio chipsets. The price for a LoRaWAN gateway typically ranges from 250 EUR to 1500 EUR. Deployment of a LoRaWAN network as private enables to cut the operation costs. The drawback is the need of having the necessary technical expertise for deploying and supporting the network's operation. The use of external MQTT broker and IoT platform is optional and should be evaluated with respect to needs of the application.

\section{CONCLUSIONS AND LESSONS LEARNED}

In the paper we detailed the design and reported the initial results coming from deployment and use of a windturbine monitoring solution based on the LoRaWAN LPWA technology. Based on our results, the following conclusions and recommendations can be given. First, LoRaWAN deployed as a private network appeared to be quite convenient and efficient for enabling connectivity of IoT sensors at isolated remote location. Second, the use of an NS integrated with the GW was quite convenient for our use case. This approach enables to both reduce the operation expenditures and increase the security. Third, the use of a third-party MQTT broker and an external IoT platform for logging the data and implementing a GUI enabled fast development of the respective functionalities. Nonetheless, this compromised the reliability of the data transfers and introduced additional delays. In future, we plan to modify this part of the system by replacing the IoT platform, and, at some phase, the external MQTT broker.

To conclude, our practical experiences show that LoRaWAN provides a decently easy-to-use and cost efficient solution, which can enable a sheer diversity of non-critical monitoring applications in the context of power grids. Even though, before making the final decision, there is one component, which we have not addressed in this paper, which is the scalability aspect. In future, we plan to investigate this aspect by the means of extensive simulations in the context of wind-pharm scenario.

\section{ACKNOWLEDGMENT}

The work was taken in the context of P2P-SMARTEST project, an Innovation Action funded by the H2020 Programme, contract number 646469, and supported by LPWAN evolution activity funded by University of Oulu, and Academy of Finland 6Genesis Flagship (grant 318927).

\section{REFERENCES}

[1] X. Fang et al., "Smart Grid - The new and improved power grid: a survey," IEEE Commun. Surv. \& Tut., vol. 14, pp. 944-980, 2012.

[2] Z. M. Fadlullah et al., "Toward intelligent machine-to-machine communications in smart grid," IEEE Commun. Mag., vol. 49, no. 4, pp. 60-65, Apr. 2011.

[3] S. W. Luan et al., "Development of a smart power meter for AMI based on ZigBee communication," in Proc. Int. Conf. Power Electron. Drive Syst., 2009, pp. 661-665.

[4] E. Spanò et al., "Last-meter Smart Grid embedded in an Internet-ofThings platform," IEEE Trans. Smart Grid, vol. 6, pp. 468-476, 2015.

[5] M. Barbiroli et al., "Smart metering wireless networks at $169 \mathrm{MHz}$," IEEE Access, vol. 5, pp. 8357-8368, 2017.

[6] J. J. Nielsen et al., "What can wireless cellular technologies do about the upcoming smart metering traffic?," IEEE Commun. Mag., vol. 53, pp. $41-47,2015$.

[7] L. Ding et al., "Distributed energy management for Smart Grids with an event-triggered communication scheme," IEEE Trans. Control Syst. Technol., in press.

[8] A. Pouttu et al., "P2P model for distributed energy trading, grid control and ICT for local smart grids," in Proc. Eur. Conf. Netw. Commun., 2017, pp. 1-6.

[9] X. Chen et al., "Real-time energy trading and future planning for fifth generation wireless communications," IEEE Wireless Commun., vol. 24, pp. 24-30, Aug. 2017.

[10] S. G. Barbosa et al., "Real-time data monitoring with Zigbee wireless transmission applied to a WECS," in Proc. Brazilian Power Electron. Conf., 2017, pp. 1-6.

[11] Y. Peng et al., "Sensor fault detection and isolation for a wireless sensor network-based remote wind turbine condition monitoring system," IEEE Trans. Ind. Appl., vol. 54, pp. 1072-1079, 2018.

[12] R. M. Zago and F. Fruett, "A low-cost solar generation monitoring system suitable for internet of things," in Proc. 2nd Int. Symp. Instrum. Syst., Circuits Transducers, 2017, pp. 1-6.

[13] P. Ashwini and R. Umamaherwari, "Wireless sensor network for condition monitoring of remote wind mill," in Proc. Int. Conf. Innovations Green Energy Healthcare Techn., 2017, pp. 1-7.

[14] R. N. Patel, "Techno-commercial analysis of wired and wireless monitoring in operational solar PV plants," in Proc. IEEE Ind. Electron. Appl. Conf., 2016, pp. 104-111.

[15] U. Raza et al., "Low power wide area networks: an overview," IEEE Commun. Surv. \& Tut., vol. 19, pp. 855-873, 2017.

[16] K. Mikhaylov and J. Petäjäjärvi, "Design and implementation of the plug\&play enabled flexible modular wireless sensor and actuator network platform", Asian J. Control, vol. 19, pp. 1-21, 2017.

[17] J. Haapola et al., "Peer-to-peer energy trading and grid control communications solutions' feasibility assessment based on key performance indicators," presented in Proc. 87th Vehicular Technol. Conf., 2018, pp. 1-5. 